\title{
TaskForce TARMED
}

Endlich können wir eine frohe Botschaft übermitteln: Die Verrechnung der Gegenseite bei Vergleichsaufnahmen paariger Extremitäten kann unter bestimmten Voraussetzungen zu Lasten der Kostenträger vorgenommen werden. Eine entsprechende Publikation des PIK-Entscheides soll demnächst erfolgen. Somit kann man nur sagen, dass die Vernunft endlich gesiegt hat. Die genauen Voraussetzungen, unter welchen eine Verrechnung erlaubt sein wird, werden auf unserer Webseite www.locomo.ch veröffentlicht, sobald der PIK-Entscheid publiziert wurde.

Noch unklar ist, wie weit der Tarif für die bildgebenden Verfahren, insbesondere die Ultraschallpositionen im Rahmen der Tarmed-Tarifrevision (Kapitel 39) Änderungen erfahren wird und ab welchem Zeitpunkt diese dann gültig werden. Es kann jedenfalls noch lange dauern...

Beat Dubs

Leiter TaskForce Tarmed 